\title{
INTRA-SCLERAL PARATHALAMUS IMPLANT: EFFICACY AND SAFETY IN RABBIT EYES
}

\author{
Efstathios T. Detorakis, ${ }^{1,2}$, Konstantina Loggaki², Nikolaos Karyotakis ${ }^{2}$, Harilaos Ginis ${ }^{2}$ \\ Department of Ophthalmology, University Hospital of Heraklion, Greece'; Institute of Vision \& Optics, University of Crete ${ }^{2}$
}

Summary: Purpose: A variety of anti-glaucomatous shunt designs have been proposed so far. This study evaluates the feasibility of a novel shunt design, the intra-scleral parathalamus implant (IPI), in a rabbit eye model. Methods: Ten healthy albino rabbits were included. Measurements of the IOP, using TonoPenXL, as well as ocular rigidity (OR) and aqueous outflow facility (AO), using a previously described methodology, were performed in both eyes of each animal. Subsequently, the IPI was implanted at the left eye of all animals whereas the right eye served as control. Measurements of IOP were repeated at weekly intervals for 2 months postoperatively whereas measurements of OR and AO were repeated at the 1st and 2nd postoperative week. Results: The IOP decreased significantly whereas the AO increased significantly postoperatively at the operated eyes. A significant postoperative decrease in OR was also recorded at the operated eyes. Respective differences at the fellow eyes were statistically not significant. Two animals developed postoperative complications and were excluded from follow-up. Conclusions: Results imply that the implantation of the IPI is feasible in rabbit eyes with significant favorable effects on the IOP, AO and OR.

Key words: Glaucoma surgery; Tube; Valve; Rabbit eye; Intraocular pressure; Sclera; Suprachoroidal space

\section{Introduction}

Anti-glaucomatous tube shunt devices are widely used in modern glaucoma surgery, especially in cases in which other procedures have failed (1-3). Current designs include valved devices (such as the Ahmed and Krupin) and nonvalved devices (such as the Molteno and Baerveldt) (1-3). In the former type there is a true valve mechanism incorporated into the implant, aimed at regulating postoperative intra-ocular pressure (IOP), thus avoiding an excess outflow of aqueous, potentially associated with postoperative ocular hypotony (1-3). Nevertheless, postoperative hypotony still remains a common complication with most currently available designs (1-3).

Most tube shunt devices consist of a plate (placed episclerally) and a tube (inserted in various intraocular positions, such as the anterior chamber, posterior chamber or vitreous cavity) (4). The latter is routinely covered with patching materials, such as cadaveric pericardium (5). However, the close proximity of the tube or plate with the conjunctiva occasionally results in conjunctival erosions, which may be difficult to treat (6). A variety of micro invasive glaucoma surgery (MIGS) implants has also been proposed (7). Although they vary considerably in design and anatomical position of insertion, most MIGS implants do not provide the hypotonic power of classic tube shunts and in many cases they serve as adjunctive anti-glaucomatous treatment during cataract surgery $(7,8)$.
Taking into account the drawbacks of episclerally placed implants we aimed at developing a new anti-glaucomatous implant, the intra-scleral parathalamus implant (IPI), which could provide adequate IOP control but would minimize the risk of postoperative hypotony and implant material exposure. This study examines the feasibility of use for IPI, in a rabbit eye model by assessing IOP, aqueous outflow facility (AO) and ocular rigidity (OR) changes following IPI implantation.

\section{Methods}

This is an experimental study on animal (rabbit) eye model. Ten (10) albino New Zealand female rabbits with an average body weight of $2.5 \mathrm{Kg}$ were included. The study design conformed to the National (Greek) Guidelines for animal usage in research.

IOP measurements were performed following topical anaesthesia with Proparacaine Hydrochloride 0.5\% (Alcaine, Alcon Laboratories, Inc., Fort Worth, TX, USA) using the Tono-PenXLtonometer (Reichert Technologies, Depew, NY,USA). The measurement technique included covering of the instrument tip with a purpose-made cup, calibration of the instrument per manufacturer instructions and gently touching the cornea with the tip of the tonometer (Figure $1 \mathrm{~A}$ ). Five readings were taken and the average value was recorded. Measurements of the IOP in both eyes of all animals were performed at least 1 week before the IPI insertion and 
at weekly intervals postoperatively for a 2-month followup period.

$\mathrm{AO}$ and $\mathrm{OR}$ measurements were performed following adequate sedation of the animals, achieved by subcutaneous administration of $1 \mathrm{ml}$ of xylazine hydrochloride (Rompun, Bayer AG, Leverkusen, Germany) and $1 \mathrm{ml}$ of ketamine Hydrochloride (Imalgene, MERIAL, Lyon, France). Measurements of both AO and OR were performed using a previously described methodology, which was developed at the Institute of Vision and Optics (IVO) of the University of Crete. Briefly, a 19G cannula is inserted into the anterior chamber at the corneoscleral limbus. The cannula is connected to an infusion system capable of delivering calibrated amounts of balanced salt solution and of manometrically recording the IOP at the same time (Figure 1B). Based on the relation between the fluid volume administered and changes in the IOP, a purpose-designed software (9) (Figure 1C) is used to calculate OR (i.e. the coefficient of the ocular pressure-ocular volume mathematical relation in $\mathrm{mmHg} / \mu \mathrm{L}$ ) and $\mathrm{AO}(\mu \mathrm{L} / \mathrm{min} / \mathrm{mmHg})$. Measurements of $\mathrm{AO}$ and OR were performed 1 week before the IPI implantation as well as on the 1-week and 2-week postoperative intervals. IOP, OR and AO score changes were statistically analyzed using the statistical package SPSS 8.0 for windows (SPSS, Chicago, IL).

\section{Technical description and mechanism of action of the IPI}

The IPI consists of a medical grade silicone tube with an internal diameter of $0.34 \mathrm{~mm}$ (inserted into the anterior chamber) and a thin plate (connected with the tube) which is formed by 2 rectangular $(3.5 \times 6.5 \mathrm{~mm})$ thin medical grade silicone sheets. The surface area of aqueous outflow is $28 \mathrm{~mm}^{2}$, corresponding to both upper and lower aspects of the plate. Many other bio-compatible materials, apart from silicone, may be used in the construction of IPI. Between the sheets, a slit space is defined which receives the aqueous (Figure 2). The plate is placed underneath a partial thickness scleral flap, which is then sutured in a water-tight fashion, thus the plate is located completely intra-sclerally (Figures 3A, 3C). Side protuberances extending from the silicone sheets, embedded into the sclera adjacent to the flap margins can stabilize the implant (Figure 2). The sheets are perforated, enabling the unobstructed outflow of aqueous into the intra-sceral space formed underneath the superficial scleral flap (Figure 2). Moreover, the inferior sheet, which is in close contact with the residual scleral bed, has micro-protuberances which become impinged into deeper sclera in close proximity with the underlying supra-choroidal space (Figure 4). However, the supra-choroidal space per se is not entered surgically, thus minimizing risks of haemorrhage or choroidal detachment. The thick superficial scleral flap implies that the layer of deeper residual sclera is accordingly thin, which may facilitate diffusion of aqueous to the supra-choroidal space. However, deep sclerectomy may additionally be performed to further re-
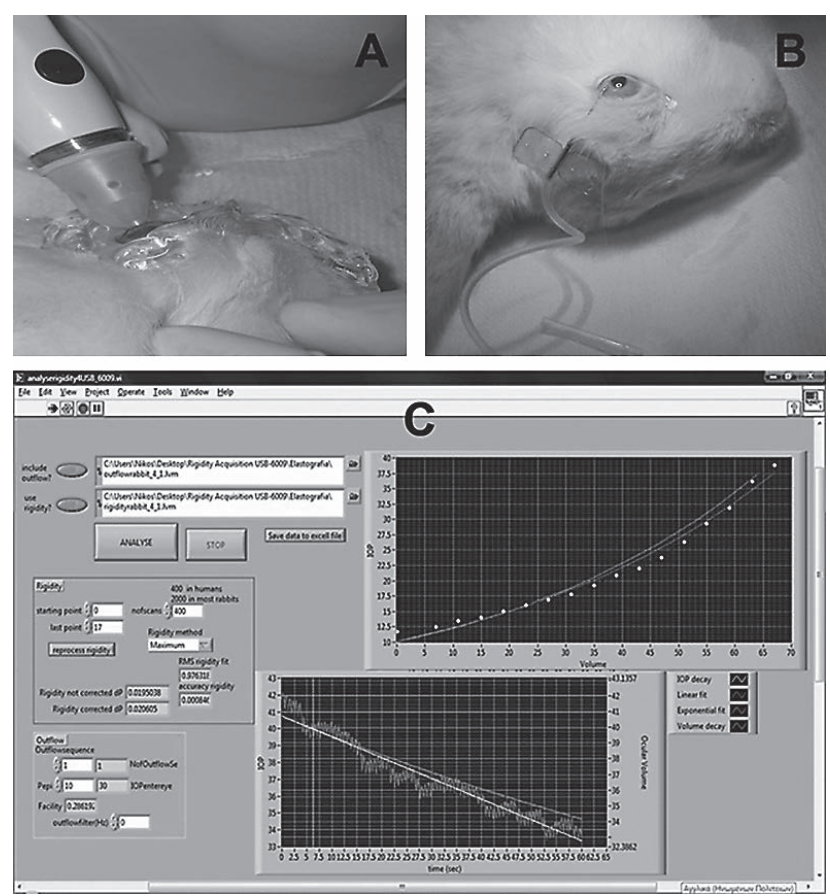

Fig. 1: Measurement of IOP using the TonoPenXL (A) and measurement of the OR and AO using a manometric catheter introduced into the anterior chamber (B) with a purpose-developed software (C).

duce the thickness of residual deeper sclera (Figure 3B). Aqueous diffusing though the residual deeper sclera into the supra-choroidal space may then exit the eye through the vortex vein system (Figure 3D). Taking into account the relatively large surface area of the scleral flap, part of

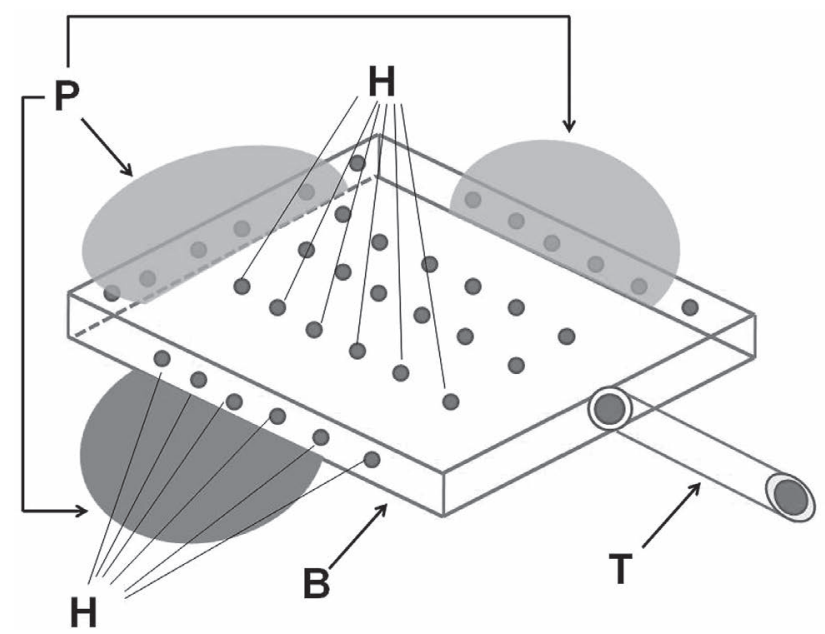

Fig. 2: IPI design. The body of the implant (B) has outflow holes $(\mathrm{H})$ and is connected with a tube $(\mathrm{T})$ to the anterior chamber, acting as a parathalamus. Side protuberances $(\mathrm{P})$ stabilize the implant intra-sclerally. 

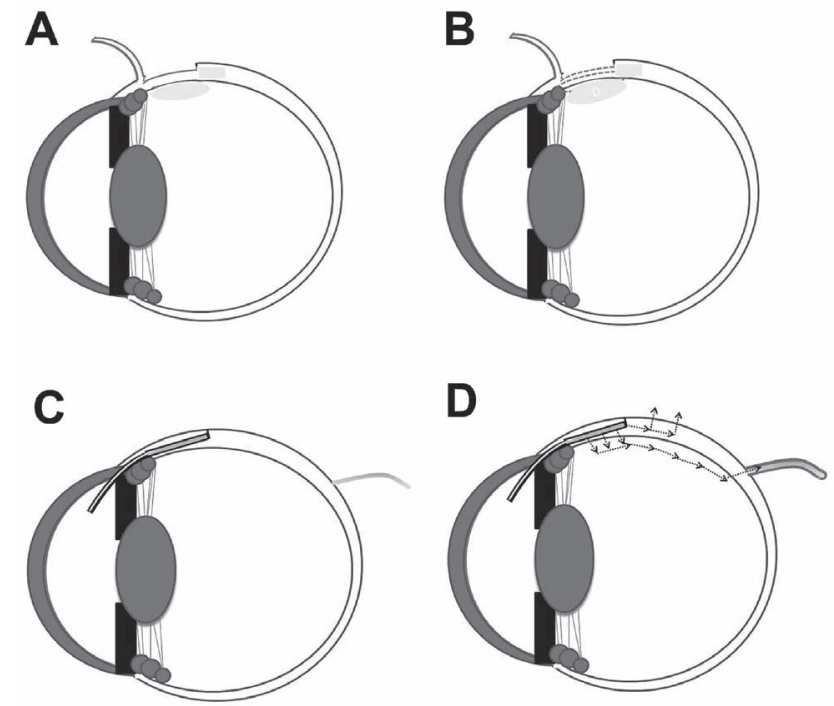

Fig. 3: Schematic presentation of IPI implantation. A partial thickness scleral flap is raised (A). Deep sclerectomy may be performed (dashed lines, B). The IPI is implanted completely intra-sclerally in a water-tight fashion (C). The outflow of aqueous may be achieved either transclerally or via the supra-choroidal space and through the vortex vein system (D).

the aqueous fluid may also exit the eye via the intra-scleral venous plexuses. The IPI is not valved but since the scleal flap is sutured in a water-tight fashion, the implant remains completely intra-ocular (intra-scleral). Thus, the IOP control may be mediated through the inherent auto-regulatory physiologic mechanisms of the eye. Moreover, the lack of

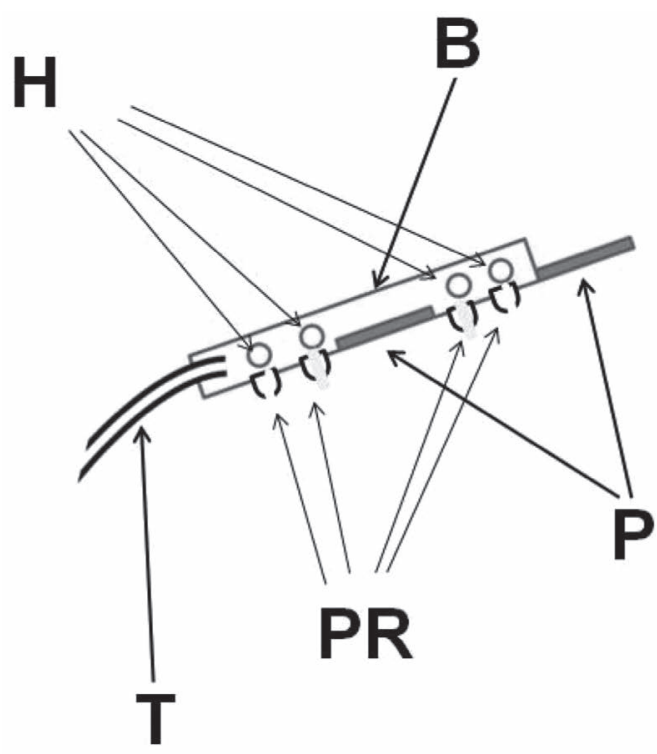

$\mathrm{H} 2$

Fig. 4: Profile view of the IPI showing the body (B), tube (T), side protuberances for intra-scleral stabilization $(\mathrm{P})$ and deep protuberances (PR) for facilitated diffusion of aqueous into the supra-choroidal space. direct contact between conjunctiva and the implant implies a reduced risk of conjunctival thinning or erosion.

\section{Surgical implantation of the IPI in rabbit eyes}

The IPI was implanted at the left eye of all animals. Following the placement of a 7.0 Vicryl traction suture to clear cornea to infra-duct the eye, a superior limbal conjunctival peritomy was performed (Figure 5A). The superior conjunctiva and Tenon's capsule were undermined and reflected to expose the superior sclera. A $7 \times 4 \mathrm{~mm}$ partial thickness scleral flap was then marked and elevated (Figure 5B). The flap was created adequately thick to leave only a thin residual layer of sclera underneath. The anterior chamber was then entered with a $23 \mathrm{G}$ needle underneath the scleral flap and the tube of the IPI was adequately trimmed in length and inserted bevel-up into the anterior chamber through the needle entrance (Figure 5C). The plate of the IPI was placed under-
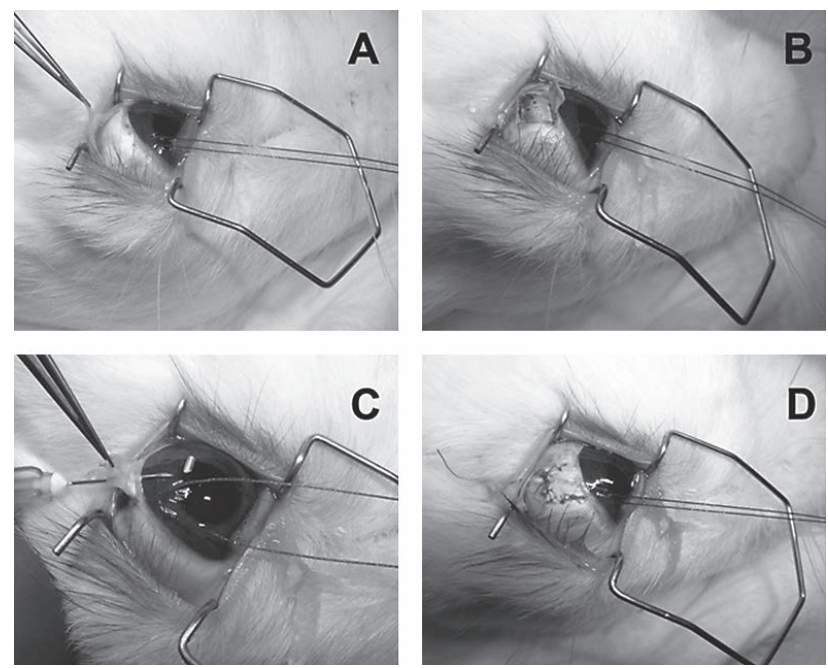

Fig. 5: Steps of IPI implantation in a rabbit eye. Conjunctival peritomy is performed (A). A sufficiently deep partial thickness scleral flap is raised, leaving a thin deeper lamella of sclera to separate the site from the supra-choroidal space (B). The anterior chamber is entered under the superficial scleral flap with a $23 \mathrm{G}$ needle parallel to the iris plane (C). The valve is implanted and the superficial scleral flap sutured in a water-tight fashion (D).
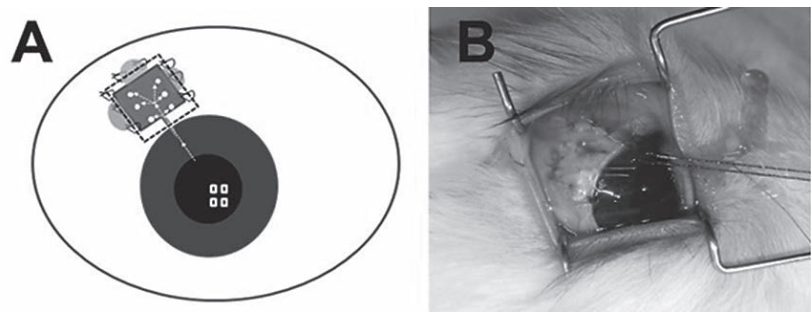

Fig. 6: Schematic anterior view of the implanted IPI (A) and respective photo of a rabbit eye implanted with an IPI (B). 
neath the superficial scleral flap which was then sutured in a water-tight fashion with several interrupted sutures (Figure 5D). The conjunctiva was then sutured to cover the flap site (Figure 6). Postoperatively, all animals received eye drops of tobramycin $(0.3 \%)$-dexametazone $(0.1 \%)$ (Tobradex, Alcon Laboratories, Inc., Fort Worth, TX, USA) qid for the first 2 postoperative weeks.

\section{Statistical analysis}

Comparisons between preoparative and postoperative scores of IOP, AO and OR were performed with paired-samples t-test. Statistical significance was set at $p<0.05$.

\section{Results}

The IPI implantation was completed without major intraoperative complications in all animals. No animal died during the operations of the follow-up period. Postoperative complications included a case of ocular infection (presumably endophthalmitis) in 1 animal and a case of shallow anterior chamber, presumably due to persistent choroidal detachment, in 1 case. Both animals with complicated procedures were excluded from follow-up and statistical analysis of data.

The postoperative IOP displayed a statistically significant reduction at the operated eyes during the first 3 postoperative weeks, compared with preoperative IOP scores (paired samples t-test score 12.23, 11.20 and 11.07, respectively for the 1 st, 2 nd and 3 rd week, $\mathrm{p} \approx 0.00$ ). Thereafter, a gradual increase and stabilization of the IOP at the operated eyes was noted. However, the IOP at the operated eyes continued to be significantly reduced, compared with preoperative readings, throughout the follow-up period.

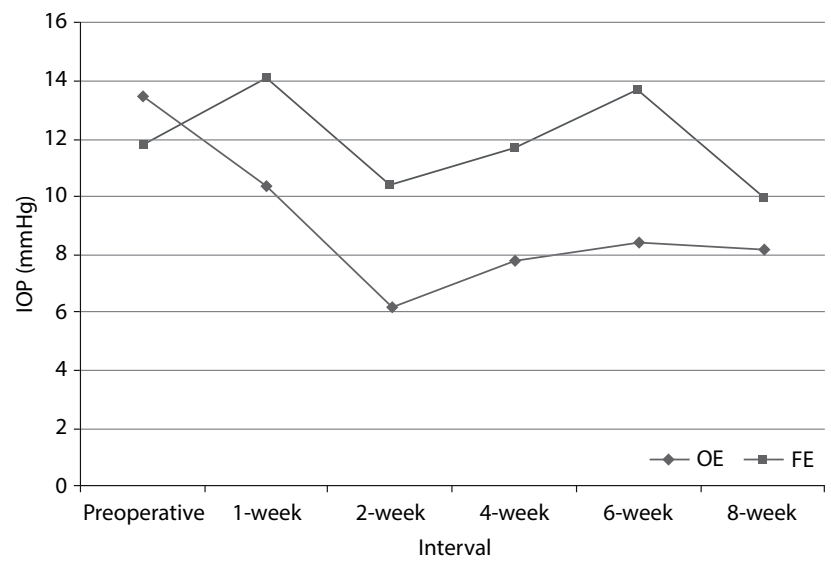

Fig. 7: Diagram of the mean IOP changes following IPI implantation for the left eye (operated eye - rhomboid dots) and right eye (fellow eye-square dots) eyes. Measurements were performed on a weekly basis from the pre-operative interval until the 2-month post-operative interval.
Interestingly, a slight (statistically not significant) reduction in the IOP at the control eyes was also noted, except for the 1st-week postoperative interval, on which the IOP at the control eyes was slightly increased, compared with preoperative levels (again, the difference with preoperative levels was statistically not significant). Mean IOP scores on all intervals for both operated (left) eyes and control (right) eyes are presented in Figure 7.

The AO and OR scores were significantly increased and reduced, respectively, at the operated eyes on both 1 -week and 2-week postoperative intervals, compared with

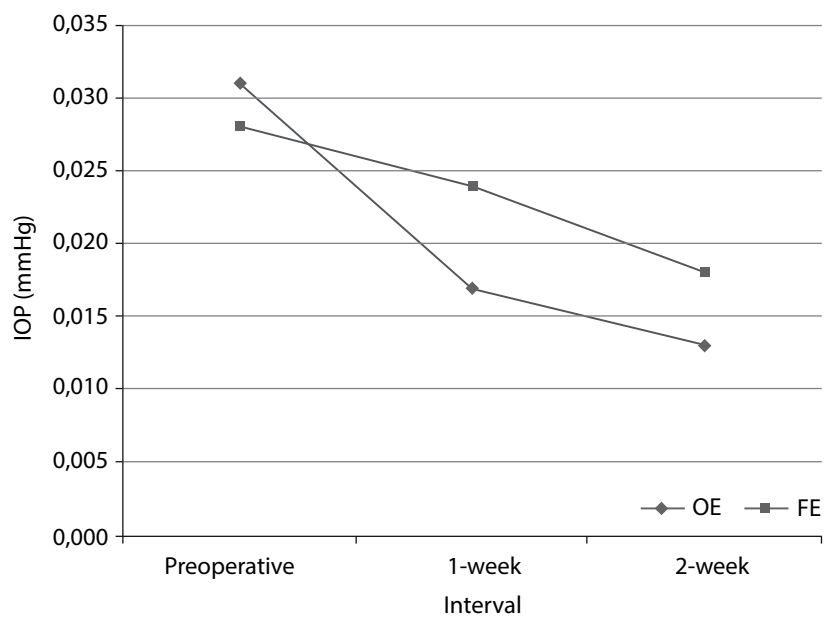

Fig. 8: Diagram of the mean OR changes following IPI implantation for the left eye (operated eye - rhomboid dots) and right eye (fellow eye - square dots) eyes. Measurements were performed 1 week preoperatively as well as 1 and 2 weeks postoperatively.

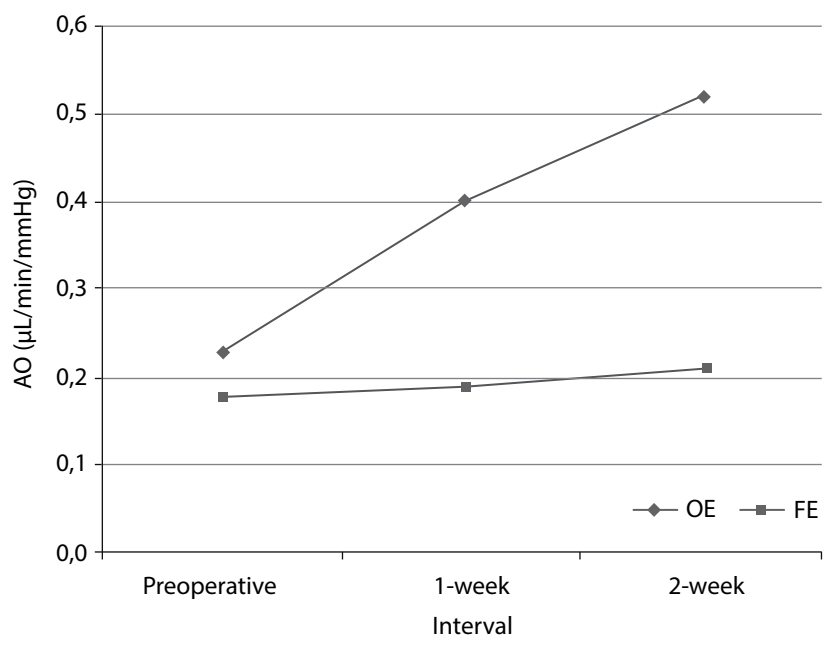

Fig. 9: Diagram of the mean AO changes following IPI implantation for the left eye (operated eye - rhomboid dots) and right eye (fellow eye - square dots) eyes. Measurements were performed 1 week preoperatively as well as 1 and 2 weeks postoperatively. 
preoperative readings (paired samples t-test score for the AO changes -7.53 and $-6.89, p=0.01$ for the 1 st and 2 nd weeks, respectively and paired samples t-test score for the OR changes 6.80 and 5.34, $p=0.01$ and $p=0.02$ for the 1 st and 2nd weeks, respectively). Respective AO and OR score changes between pre-operative and postoperative intervals for the control eyes were statistically not significant. Mean $\mathrm{OR}$ and AO scores during the follow-up period are presented in Figures 8 and 9, respectively.

\section{Discussion}

This is a feasibility study of a new anti-glaucomatous implant design, the IPI, in rabbit eyes. The IPI differs from other anti-glaucomatous implant designs in that it is implanted completely intra-sclerally, creating a permanent parathalamus between the sheets of its plate, i.e. an intra-scleral space in communication with the anterior chamber through the valve tube. The mechanism of action of IPI relies on the creation of an intra-scleral parathalamus cavity to receive the aqueous (10). The intra-scleral implantation of the IPI plate theoretically dictates a permanent presence of an intra-scleral cavity. The outflow of aqueous may be performed via either intra-scleral venous plexuses (and possibly trans-scleral diffusion through thin sclera (11) or via the supra-choroidal space and the vortex vein system. In this respect, the IPI resembles the Solx Gold micro-shunt (SOLX Inc. Waltham, MA, USA), which is designed to establish a direct communication between the anterior chamber and the supra-choroidal space (12). However, in contrast with the Solx Gold implant, in the case of IPI the supra-choroidal space is not surgically entered, thus theoretically reducing the risk of supra-choroidal haemorrhage or detachment. The IPI is a non-valved implant operating as a closed system within the eye, which enhances the inherent physiological mechanisms of alternative aqueous outflow and relies on IOP auto-regulation to avoid postoperative ocular hypotony. The lack of bleb formation and lack of direct contact with the conjunctiva also imply a potentially reduced incidence of ocular tissue erosions and exposure of the implant.

Although rabbit eyes differ from human eyes from various histological and physiological standpoints (13), they were chosen as the animal eye model to test IPI in this study due to easy manipulation and availability. Several previous studies on anti-glaucomatous valves have also employed rabbit eyes to assess efficacy and safety parameters (14-16). The sustained decrease in IOP scores and increase in AO detected on all postoperative intervals at the operated eyes, compared with preoperative IOP scores, implies that the IPI is effective in reducing the IOP. The gradual increase of IOP at the operated eyes after the 3rd postoperative week possibly reflects local scarring or the resolution of any amounts of postoperative exudative choroidal detachment. However, the fact that the IOP after the 3rd postoperative week stabilized at significantly lower levels, compared with preoperative IOP scores, implies that the IPI remained active throughout the follow-up period. The increase in AO recorded is in accordance with an effective function of IPI at the operated eyes.

Interestingly, IOP changes were also detected at the control (non-operated) eyes, although at a statistically not significant level. Previous studies in animal and human eyes have also detected IOP changes at the fellow (non-operated) eyes following anti-glaucomatous procedures in one eye (consensual ophthalmotonic response, COR) (17-19). Although several mechanisms have been proposed to explain COR in human eyes, it may be attributed to central reflex changes in ciliary body innervation, triggered by the sudden drop of IOP at the operated eyes (20). Such mechanisms may possibly explain the changes in the IOP observed in this study. Moreover, the IPI may also have other patho-physiological effects, as suggested by the decrease in OR detected at the operated eyes during the follow-up period. OR refers to the mathematical relation between the intraocular volume and pressure (expressed by an OR coefficient) and it is considered an important index of the bio-mechanical behaviour of the ocular tissues (21). Changes in OR may play a role in the pathogenesis of glaucoma through alteration in scleral elastic properties (especially in the area of the lamina cribrosa) in different IOP levels (22). An increase in the OR coefficient after prolonged IOP increase has also been documented in animal studies, in rabbits (24). Accordingly, the reduction in OR observed following IPI implantation in the present study may represent a favorable effect of IPI implantation. Alterations in choroidal circulation or ocular wall structure may explain such OR changes, which have also been reported following other anti-glaucomatous procedures $(21,22)$. Although traditionally AO has been assessed in vivo using tonography or fluorophotometry, the manometric assessment with the methodology described in this study has also resulted in valid results (23). The fact that AO was significantly increased following the implantation of IPI implies that IPI is effective in producing measurable effects on AO.

Two animals were excluded from follow-up in this study due to complications associated with the IPI implantation procedure. Previous studies on rabbits evaluating experimental implant designs have also reported similar incidences of complications $(23,24)$. However, the IPI was well tolerated in the majority $(80 \%)$ of the animal eyes included, implying an acceptable safety profile in this animal population. Moreover, improvements in the geometric characteristics of the implant, including the physical dimensions of the plate or the diameter of micropores and tube (which were originally designed for human eyes) may be made in future related animal studies to better comply with the physical dimensions of the eyes included. The bio-compatibility of the implant may also be enhanced by the selection of adequate materials, including acrylic, silicone or PMMA polymers.

Limitations of the present study, apart from physiologic or clinical differences between the rabbit eye and human 
eye models, include the fact that measurements of AO and OR were performed in an invasive manner (manometric catheter introduction into the anterior chamber), which may have affected postoperative measurements, as well as the fact that the rabbit eyes included were non-glaucomatous. Accordingly, the IPI could be tested on animal eyes following the development of an experimental glaucoma model. Moreover, the histological examination of rabbit eyes with IPI, which would enable the evaluation of material biocompatibility and tissue reaction was not performed in this study, since the study design focused more on the IOP reduction outcomes as a pilot feasibility protocol. Taking into account the encouraging results, a biocompatibility study with histological examinations in rabbit eyes could follow. IPI performance in human blind glaucomatous eyes could then be performed as a next step in the future.

\section{Conflict of interest}

Efstathios T. Detorakis, MD, PhD, holds patent rights for the IPI design.

\section{References}

1. Sharaawy T, Bhartiya S. Surgical management of glaucoma: evolving paradigms. Indian J Ophthalmol 2011; 59 Suppl: S123-30.

2. Francis BA, Singh K, Lin SC, et al. Novel Glaucoma procedures: a report by the American Academy of Ophthalmology. Ophthalmology 2011; 118: 1466-80.

3. Patel S, Pasquale LR. Glaucoma drainage devices: a review of the past, present, and future. Semin Ophthalmol 2010; 25: 265-70.

4. Hitchings RA, Joseph NH, Sherwood MB, Lattimer J, Miller M. Use of one-piece valved tube and variable surface area explant or glaucoma drainage surgery. Ophthalmology 1987; 94: 1079-84.

5. Raviv T, Greenfield DS, Liebmann JM, Sidoti PA, Ishikawa H, Ritch R. Pericardial patch grafts in glaucoma implant surgery. J Glaucoma 1998; 7: 27-32.
6. Sarkisian SR Jr. Tube shunt complications and their prevention. Curr Opin Ophthalmol 2009; 20: 126-30.

7. Saheb H, Ahmed II. Micro-invasive glaucoma surgery: current perspectives and future directions. Curr Opin Ophthalmol 2012; 23: 96-104.

8. Augustinus CJ, Zeyen T. The effect of phacoemulsification and combined phaco/ glaucoma procedures on the intraocular pressure in open-angle glaucoma. A review of the literature. Bull Soc Belge Ophtalmol 2012; 320: 51-66.

9. Pallikaris IG, Kymionis GD, Ginis HS, Kounis GA, Tsilimbaris MK. Ocular rigidity in living human eyes. Invest Ophthalmol Vis Sci 2005; 46: 409-14.

10. Roy S, Mermoud A. Deep sclerectomy. Dev Ophthalmol 2012; 50: 29-36.

11. Hämäläinen KM, Kananen K Auriola S, Kontturi K Urtti A. Characterization of paracellular and aqueous penetration routes in cornea, conjunctiva, and sclera. Invest Ophthalmol Vis Sci 1997; 38: 627-34.

12. Francis BA, Singh K, Lin SC, et al. Novel glaucoma procedures: a report by the American Academy of Ophthalmology. Ophthalmology 2011; 118: 1466-80.

13. Werner L, Chew J, Mamalis N. Experimental evaluation of ophthalmic devices and solutions using rabbit models. Vet Ophthalmol 2006; 9: 281-91.

14. Löbler M, Sternberg K, Stachs O, et al. Polymers and drugs suitable for the development of a drug delivery drainage system in glaucoma surgery. J Biomed Mater Res B Appl Biomater 2011; 97: 388-95.

15. Nyska A, Glovinsky Y, Belkin M, Epstein Y. Biocompatibility of the Ex-PRESS miniature glaucoma drainage implant. J Glaucoma 2003; 12: 275-80.

16. Wilcox MJ, Barad JP, Wilcox CC, Peebles EL, Minckler DS. Performance of a new, low-volume, high-surface area aqueous shunt in normal rabbit eyes. J Glaucoma 2000; 9: 74-82.

17. Diestelhorst M, Krieglstein G. The effect of trabeculectomy on the aqueous humor flow of the unoperated fellow eye. Graefes Arch Clin Exp Ophthalmol 1991; 229: 274-276.

18. Yarangümeli A, Köz OG, Kural G. The effect of trabeculectomy on the intraocular pressure of the unoperated fellow eye. J Glaucoma 2003; 12: 108-113.

19. Vysniauskiene I, Shaarawy T, Flammer J, Haefliger IO. Intraocular pressure changes in the contralateral eye after trabeculectomy with mitomycin C. Br J Ophthalmol 2005 Jul; 89: 809-811.

20. Detorakis ET, Tsiklis N, Pallikaris IG, Tsilimbaris MK.Changes in the intraocular pressure of fellow untreated eyes following uncomplicated trabeculectomy. Ophthalmic Surg Lasers Imaging. 2011; 42: 138-43.

21. Davison PF, Galbavy EJ. Connective tissue remodeling in corneal and scleral wounds. Invest Ophthalmol Vis Sci 1986; 27: 1478-1484.

22. Usui S, Ikuno Y, Uematsu S, Morimoto Y, Yasuno Y, Otori Y. Changes in axial length and choroidal thickness after intraocular pressure reduction resulting from trabeculectomy. Clin Ophthalmol 2013; 7: 1155-61.

23. Kugelberg M, Shafiei K, Zetterström C. Single-piece AcrySof in the newborn rabbit eye. J Cataract Refract Surg 2004; 30: 1345-50.

24. Brodbelt DC, Blissitt KJ, Hammond RA, et al. The risk of death: the confidential enquiry into perioperative small animal fatalities. Vet Anaesth Analg 2008; 35: $365-73$

Corresponding author:

Accepted in revised form: 28/11/2014

Efstathios T. Detorakis, MD, PhD, Department of Ophthalmology, University Hospital of Heraklion, 71110, Heraklion, Crete, Greece; e-mail: detorakis@hotmail.com 